Durch eine Minimumbetrachtung leitet er zunächst mittelst Variation von $U$ allein einen Werth für $A \cdot \varepsilon$ ab, der aber nur eine statische Bedeutung haben kann, den jedoch Lorenzoni beibehält. Während dieser nun nach dem
Satz von der Erhaltung der lebendigen Kraft die Gleichung $\mathrm{d}(E+U): \mathrm{d} t=0$ bildet, leitet Peirce nach dem Hamilton'schen Princip zwei Differentialgleichungen $a b$, aus denen er meine Werthe von $\Delta$ und $L^{\prime}$ hätte finden muissen.

Potsdam, April 1897.

Helmert.

\title{
Ueber die Benutzung der Projectionsfactoren \\ bei Untersuchung der systematischen Fehler heliometrischer Distanzmessungen.
}

Von H. Battermann.

Herr Dr. Cohn spricht A. N. 341 I die Ansicht aus, dass meine Bemerkungen über die Unsicherheit der Projectionsfactoren (A. N. 3406) sich nur auf die Bestimmung des Scalenwerthes, aber nicht auf die Bestimmung systematischer Fehler beziehen könnten. Diese Unsicherheit müsse als die zufälligen Messungsfehler vergrössernd angesehen werden und lasse sich nicht umgehen.

Ich glaube nun genügend darauf hingewiesen zu haben, dass die Unsicherheit der Projectionsfactoren allerdings nur in dem Falle unmerklich wird, wenn jene Factoren aus scharfen Positionswinkelmessungen an Heliometern bestimmt sind, oder wenn die Sterne des Bogens sehr nahe in einem grössten Kreise liegen. Sind diese Bedingungen nicht erfuillt, so lässt sich thatsächlich die Unsicherheit der Meridianpositionen, aus welchen die Factoren abgeleitet sind, in keiner Weise umgehen; aber die daraus hervorgehende Unsicherheit ist durchaus systematischer Natur und kann durch die Zahl der Heliometermessungen nicht verringert werden. Wenn man Anspruch darauf erhebt, dass das Resultat der Untersuchung eine reale Bedeutung haben und nicht nur ein rechnungsmässiges sein soll, so muss man entweder die Meridianpositionen selbst vermittelst der aufzustellenden Gleichungen zu verbessern suchen, und zwar im Wesentlichen die Coordinaten senkrecht zu dem betrachteten Bogen; oder, wenn hierfür das Material nicht ausreicht, sollte man den mittleren Fehler der einzelnen Gleichungen, soweit er von der Unsicherheit der Meridianpositionen herrubrt, angeben. Dies betrifft in gleicher Weise Bestimmungen des Scalenwerthes und Bestimmungen systematischer Fehler.

Ich habe nun vorgeschlagen, die Projectionsfactoren ganz zu vermeiden, weil diese die Berechnung nur scheinbar

$$
(a f)=\Sigma \text { Hel. }+\boldsymbol{\Sigma} \text { Corr }+(\text { Mer }
$$

$\operatorname{Mer}_{(a f)}-\mathcal{L}$ Mer. ist die Reduction der Summe der Partialdistanzen auf die Hauptdistanz «, aber abgleitet aus den Meridianpositionen; diese aus den Meridianpositionen leicht 24 berechnende Reduction ersetzt hier die Projectionsfactoren. $\Delta_{(a f)}-\Sigma \Delta$ giebt den Einfluss der Unsicherheit der Meridianpositionen an. erleichtern, thatsächlich vielmehr, wenn man nämlich den Einfluss der Meridianpositionen untersuchen will, compliciren und undurchsichtig machen. Ich schlug vor, die Summe der heliometrisch bestimmten Distanzen mit der Sunme der aus den Meridianpositionen abgeleiteten Distanzen zu vergleichen. Thatsächlich sind natürlich beide Methoden, wenigstens bis auf kleine, meist unwesentliche Glieder, bei gehöriger Entwickelung identisch. Aber in der von mir vorgeschlagenen, ubrigens nicht neuen Weise sind die Ent. wickelungen einfacher. Ich will dies an einem Beispiel dar. legen, und wähle den Hydrabogen für Epoche r885.o. Die Grundlagen sind in "Venusdurchgänge 1874 und 1882 ", Bd.V S. $3^{61}$ u. $3^{62}$ angegeben.

Bezeichnet $\boldsymbol{\Sigma}$ Hel. die Summe der fünf heliometrisch gemessenen Distanzen $a b \ldots$ ef, $\Sigma$ Corr. die Summe der systematischen Correctionen, welche an diese anzubringen sind; ferner $\Sigma$ Mer. die Summe der entsprechenden, aber aus den Meridianpositionen berechneten Distanzen, $\boldsymbol{\Sigma} \Delta$ die Summe der Verbesserungen, welche letztere wegen Verbesserung der Meridianpositionen erfordern, so hat man die Gleichung :

$$
\boldsymbol{\Sigma} \text { Hel. }+\boldsymbol{\Sigma} \text { Corr }=\boldsymbol{\Sigma} \text { Mer. }+\boldsymbol{\Sigma} \boldsymbol{d}
$$

Wenn ferner für die Hauptdistanz af der aus den Meridianpositionen abgeleitete Werth mit $\operatorname{Mer}_{(a f)}$, die entsprechende Verbesserung wegen Verbesserung der Meridianpositionen mit $\Delta_{(a f)}$, endlich dèr wahre Werth mit $(a f)$ bezeichnet wird, so ist

$$
(a f)=\operatorname{Mer}_{(a f)}+\Delta_{(a f)} .
$$

Durch Subtraction der ersten Gleichung von der zweiten und passende Vereinigung der Glieder erhält man

$$
\left.r_{(a f)}-\Sigma \text { Mer }\right)+(\Delta(a f)-\Sigma \Delta) \text {. }
$$

Für den Hydrabogen erhält man aus den citirten Angaben ohne Weiteres, wenn die Indices $1 \ldots 6$ sich auf die Sterne $a \ldots f$ beziehen, und wenn, der grösseren Homogeneität wegen, $\Delta^{\prime} \alpha_{1}\left(=10 \Delta \alpha_{1}\right)$ die Verbesserung von $\alpha_{1}$ in Einheiten von $0^{5}, 1, \Delta \delta_{1}$ dagegen die Verbesserung von $\delta_{1}$ in Einheiten von 's" bedeutet:

$$
\begin{aligned}
(a f)=\Sigma \text { Hel. }+\Sigma \text { Corr }-188.631 & +0.245 \Delta^{\prime} \alpha_{1}-0.356 \Delta^{\prime} \alpha_{2}+0.118 \Delta^{\prime} \alpha_{3}-0.291 \Delta^{\prime} \alpha_{4}+0.590 \Delta^{\prime} \alpha_{5}-0.306 \Delta^{\prime} \alpha_{6} \\
& +0.039 \Delta \delta_{1}-0.067 \Delta \delta_{2}+0.029 \Delta \delta_{3}-0.087 \Delta \delta_{4}+0.130 \Delta \delta_{5}-0.044 \Delta \delta_{6}
\end{aligned}
$$

Diese Gleichung reducirt die Summe der fünf Partialdistanzen $a b \ldots$ ef auf den uns noch unbekannten Werth $(a f)$ der Hauptdistanz. Auf den gleichen Werth $(a f)$ kann man ebenso jede andere Combination von Partialdistanzen, 
welche die Endpunkte von af geschlossen verbindet, reduciren; man erhält so analoge Gleichungen für $(a f)$. Durch Subtraction dieser verschiedenen Gleichungen von einander wird die Unbekannte $(a f)$ eliminirt; man erhält Gleichungen zwischen den Messungsresultaten, den systematischen Correctionen der Heliometermessungen und den Verbesserungen der Meridianpositionen. Bezuiglich der letzteren kommen hier, d. h. bei einem Declinationsbogen, ganz vorwiegend die Verbesserungen der Rectascensionen in Betracht. Entweder müssten daher neben den systematischen Correctionen auch diese Verbesserungen der Rectascensionen abgeleitet werden; oder aber, wenn man das nicht will, muss auf Grund der mittleren Fehler der Meridianpositionen der hieraus stam. mende mittlere Fehler der resultirenden Gleichungen berechnet werden. Aus den 1. c. angegebenen mittleren Fehlern der Rectascensionen und Declinationen der sechs Sterne und der bez. Eigenbewegungen erhielt ich als mittleren Fehler der obigen Gleichung (jedoch für andere Epoche):

$$
\begin{aligned}
& \text { Epoche i } 890 \text { : Von } \varepsilon_{\alpha} \text { herriuhrend } \pm 0.1 \mathrm{I} \text {, von } \varepsilon_{\delta} \text { herrührend } \pm 0.03 \text {, zusammen } \pm 0.11
\end{aligned}
$$

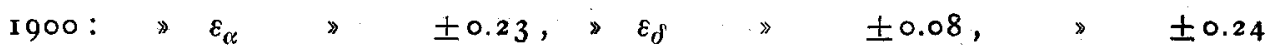

Da die Unsicherheit der Meridianpositionen ihren grössten Einfluss auf die hier behandelte Summe der fünf kleinsten Distanzen ausüben wird, so darf man schliessen, dass aus Beobachtungen zur Epoche 1890 jedenfalls noch eine Ableitung der systematischen Fehler der Heliometermessungen möglich sein wird. Zur Epoche I 900 würde die Unsicherheit der alten Meridianpositionen das Resultat schon etwas zweifelhaft machen, besonders, wenn man vielleicht annehmen darf, dass die Unsicherheit der Rectascensionen thatsächlich, z. B. in Folge der Helligkeitsgleichung, grösser ist, als sie sich rechnungsmässig ergeben hat.

$$
\begin{aligned}
(a f)=\Sigma \text { Proj. }+\Sigma \text { Corr. } & +0.246 \Delta^{\prime} \alpha_{1}-0.357 \Delta^{\prime} \alpha_{2}+0.1 \mathrm{I} 9 \Delta^{\prime} \alpha_{3}-0.277 \Delta^{\prime} \alpha_{4}+0.576 \Delta^{\prime} \alpha_{5}-0.307 \Delta^{\prime} \alpha_{6} \\
& +0.024 \Delta \delta_{1}-0.056 \Delta \delta_{2}+0.033 \Delta \delta_{3}-0.105 \Delta \delta_{4}+0.126 \Delta \delta_{5}-0.022 \Delta \delta_{6}
\end{aligned}
$$

Die geringfügigen Differenzen gegen die frühere Gleichung entstehen durch die verschiedene Grösse der einzelnen Projectionsfactoren. Ebenso sind wieder die analogen Gleichungen für die übrigen Combinationen aufzustellen; aus den Differenzen der Gleichungen verschwindet wieder die Un.

Berlin 1897 April I5.
Bei Anwendung der Projectionsfactoren kommt man auf fast identische Gleichungen; aber die Rechnung ist etwas weniger einfach, sogar nachdem die umständliche $\mathrm{Be}$ rechnung der Projectionsfactoren ausgefuhrt ist. Man hat die einzelnen Projectionen zu berechnen, und dazu die einzelnen Aenderungen dieser Projectionen, welche den l. c. angegebenen Differentialquotienten der Logarithmen der Projections. factoren entsprechen. So erhält man für die Summe der Projectionen der Distanzen $a b \ldots$ ef auf $a f$ schliesslich die Gleichung:

\title{
Ueber Vermeidung von Zeichenfehlern bei Vorausberechnung von Finsternissen und Sternbedeckungen.
}

\author{
Von H. Battermann.
}

Bei Vorausberechnung von Finsternisserscheinungen werden, wie die' Erfahrung lehrt, häufig Zeichenfehler gemacht; dieselben betreffen meistens das Glied

$$
-\frac{m \cos (M-N)}{n}
$$

in dem Ausdruck für $T-T_{0}\left(T_{0}\right.$ sei die Zeit der Conjunction in Rectascension, $T$ die Zeit eines Contactes, resp. von Eintritt oder Austritt; alles in mittlerer Zeit des Meridians der angewandten Ephemeride). Da derartige Fehler selbst in den Angaben der Hauptephemeriden trotz doppelter Berechnung vorkommen, so dürfte es nicht überflüssig sein, auf eine allerdings auf der Hand liegende Controle hinzuweisen, welche diese Fehler unmöglich macht.

Die bekannte Gleichung für $T-T_{0}$ ist nur aus der bekannten Fundamentalgleichung der Finsternisse

$$
\begin{array}{cl}
(x-\xi)^{2}+(y-\eta)^{2}=L^{2} & \text { oder Berliner Jahrbuches : } \\
{\left[\left(T-T_{0}\right) p^{\prime}-u-\left(T-T_{0}-\tau\right) u^{\prime}\right]^{2}+\left[q_{0}+\left(T-T_{0}\right) q^{\prime}-v-\left(T-T_{0}-\tau\right) v^{\prime}\right]^{2}=k_{2} .}
\end{array}
$$

abgeleitet. $x$ und $y$ sind die bez. Coordinaten des Mondes, $\xi$ und $\eta$ diejenigen des Beobachtungsortes in einem Coordinatensystem, dessen $z$-Axe der Schattenaxe parallel ist; $L$ ist der Radius des Schattenkreises in einer durch den Beobachtungsort senkrecht zur $z$-Axe gelegten Ebene. Diese Gleichung muss daher für die gefundene Zeit $T$ des Contactes streng erfüllt sein. Also die einfache und sichere Controlle ist: man berechne $x, y, \xi, \eta, L$ unter Benutzung der schon berechneten oder in den Ephemeriden gegebenen Differentialquotienten für die gefundene Zeit des Contactes, und bilde die Summe der Quadrate $(x-\xi)^{2}+(y-\eta)^{2}$, am bequemsten unter Benutzung von Quadrattafeln; diese Summe muss $=L^{2}$ sein.

Speciell für Sternbedeckungen lautet die Controle, unter Anwendung der Bezeichnungen des Nautical Almanac

\section{H. Battermann.}

